\title{
Understanding Patient Engagement in Outpatient Cardiac Rehabilitation Programs through the Model of Therapeutic Engagement
}

\author{
Sepideh Jahandideh ${ }^{1 *}$, Elizabeth Kendall ${ }^{1}$, Samantha Low choy ${ }^{2}$, Kenneth Donald ${ }^{1}$ and Rohan Jayasinghe ${ }^{3}$ \\ ${ }^{1}$ Griffith University, Australia \\ ${ }^{2}$ Griffith Social and Behavioral Research College, Australia \\ ${ }^{3}$ Gold Coast University Hospital, Australia
}

*Corresponding author: Sepideh Jahandideh, School of Medicine \& Menzies Health Institute Queensland, Gold Coast Campus, Griffith University, Queensland, Australia

Submission: 眥February 02, 2018; Published: 眥 February 27, 2018

\section{Letter to Editor}

Patient engagement in the cardiac rehabilitation (CR) process is increasingly being viewed as essential to achieving desired clinical outcomes [1-3]. Although the topic of patient engagement has long been researched within the context of psychotherapy and mental health [3-6], research directly exploring this topic within the context of CR has only recently emerged. Most of the literature on this topic focuses on only single-variable data to predict patient engagement. Hence, there is an absence of coherent analysis of patient engagement in CR. More complex models of engagement have been developed in the context of brain injury rehabilitation $[7,8]$. These models emphasise the personenvironment interaction and acknowledge the multi-layered nature of the engagement process. The most comprehensive model of therapeutic engagement was developed by Lequerica \& Kortte [9]. In this model, the construct of therapeutic engagement is explicitly defined by identifying a series of sub-models that explain the intention to engage in rehabilitation, preparation for engagement in rehabilitation, initiation of rehabilitation, and rehabilitation evaluation and maintenance. Little research has investigated this multi-layered model to determine its utility in the context of CR. This review provides an in-depth assessment needed to create a useful framework for patient engagement in CR.

\section{The issues}

Interest in process variables has continued over time, with the main goal of identifying factors that facilitate or hinder participation in rehabilitation. Unitary variables have been identified and studied in disparate ways. For instance, researchers have focused on the way in which a patient's knowledge about rehabilitation and goals facilitates participation [10,11]. A large body of research has focused on self-efficacy and outcome expectancies as predictors of the degree to which people follow the instructions of their healthcare providers $[12,14]$. Patient motivation has also received attention in the medical literature as an important construct that can partially explain patient participation in rehabilitation $[15,16]$.

These studies only account for a small proportion of the variance in both participation and outcomes. There is a need to evaluate the complexities of the rehabilitation process to better understand and identify the active ingredients that facilitate engagement. Recently, researchers have begun to develop a more holistic conceptualization of participation in the rehabilitation process. An important construct that has emerged from this work is rehabilitation engagement [1719]. Rehabilitation engagement has been described as "a construct that captures multiple elements, including the patient's attitude toward the therapy, level of understanding or acknowledgment of a need for treatment, level of active participation in the therapy activities, and level of attendance across the rehabilitation program" [17]. By conceptualizing engagement in these concrete terms, it will become possible to more fully understand this complex process. Given that therapeutic engagement seems to encapsulate multiple components, the development of multivariate model is an important, yet challenging, endeavour. Lequerica, Kortte [9] have presented a theoretical model of therapeutic engagement which clarifies how and why patients engage in medical rehabilitation. Although the components of this theoretical engagement model can be derived from previous literature, the model has not been tested as a whole in any rehabilitation population. Thus, its applicability to cardiac patients remains unknown. This model has to form the basis of the current studies.

The model of therapeutic engagement in rehabilitation that underpins the current study places active patient involvement at the centre of successful rehabilitation experiences. If engagement is a significant factor affecting outcomes of rehabilitation, then transparency about how this process occurs is vital [20]. Research conducted to date has focused on single-variable models that are associated with motivation for, participation in, or attendance 
at rehabilitation. Moreover, the focus of research has been on individual-level factors, such as self-efficacy, without any attention to the broad social determinants of behaviour. Developing a comprehensive, multivariate Model of Therapeutic Engagement (involving looking of the relationships among a range of individual, social, and environmental variables and patients' engagement in CR programs) will drive a systematic, data-driven approach. Embedding this model into a broader socio-environmental context will provide high-quality data for researchers, healthcare providers, and rehabilitation specialists, allowing these stakeholders to formulate better engagement cost-effective interventions for short and long term engagement in CR programs. Testing this model of engagement will raise some analytical challenges in that the samples will change slightly as each subset within the model is examined.

\section{These analytical challenges include:}

A. Identifying patient attendance in CR.

B. Determining which patients will initiate contact after committing to attend CR.

\section{Assessing CR attendance fluctuation}

D. Evaluating CR program completion.

E. Monitoring CR engagement post-discharge.

At each point of analysis, the number of individuals in each subset may be different. This can lead to a large percentage of missing data fields, which would then warrant excessive use of estimation or imputation. Such an event would result in a limitedpower study. Therefore, a modular analytical approach can be used where a series of variance-weighted linear regressions (WLR) can be fitted to different subsets of the data according to each stage of the theoretical engagement model. However, it is not optimal to use a series of WLRs as this will increase the likelihood of error and will fail to provide an overall understanding of engagement, instead explicating only on each sub-stage of the model. Thus, the best explanation of patient engagement can be achieved by applying several alternative methods, including:

\section{A. Artificial neural network modelling (ANN). \\ B. Multivariate adaptive regression splines (MARS). \\ C. Structural equation modelling (SEM).}

The SEM approach could result in good explanatory performance, the ANN good predictive performance, but the MARS approach may result in both explanatory and predictive performance. The models can be chosen because, unlike basic linear regression, they can accommodate non-linear relationships between input and output features. We expect non-linearity because of the nature of the parameters affecting human behaviour and decisions regarding engagement in CR. Thus, non-linear methods can be applied in conjunction with other approaches, including WLR. None of these modelling approaches have previously been introduced into the context of CR. Comparing the performance of these three models enables us to identify the solution that most explains or predicts patient engagement in CR.

\section{Conclusion}

Patient engagement is vital to successfully manage cardiac diseases. Indicators of patient engagement can be observed where the patient and environment interface [9]. Therefore, there are many contributing variables that could influence patient engagement. There is a scarcity of research that has examined rehabilitation engagement in-depth to better understand the component process contributing to behavioural outcomes. No clinical decision support model currently exists to alert patients as well as health care providers to the factors that influence non-engagement. The Model of Therapeutic Engagement [9] can be applied to comprehensively examine the factors that impact upon the different stages of patient engagement in outpatient CR programs. The utility of four different modelling approaches (including multiple logistic regressions, structural equation modelling, multivariate adaptive regression splines and artificial neural networks) can be investigated. Each modelling approach can describe and examine the complex relationships between inputs and outputs, and uncover the underlying patterns in the data related to patient engagement. The most important issue is finding a comprehensive framework for predicting patient engagement in patients by a set of known variables. The explanatory findings could enable health care providers to identify management "levers" that can be altered to improve the chance of engagement. The predictive findings could enable health care providers to individualize a care management plan based on the predicted chance of a patient engaging (or not) in outpatient CR programs. These two objectives will be achieved by testing the Lequerica [17], Kortte [18] conceptual model using statistical and machine learning models, with benefits ranging from explanation to prediction: SEM, MARS, and ANN.

\section{References}

1. Simmons-Mackie N, Kovarsky D (2009) Engagement in clinical interaction: an introduction. Semin Speech Lang 30(1): 5-10.

2. Kayes NM, McPherson KM (2012) Human technologies in rehabilitation: 'Who'and 'How'we are with our clients. Disabil Rehabil 34(22): $1907-$ 1911.

3. King G, Currie M, Petersen P (2014) Child and parent engagement in the mental health intervention process: A motivational framework. Child and Adolescent Mental Health 19(1): 2-8.

4. Kim H, Munson MR, McKay MM (2012) Engagement in mental health treatment among adolescents and young adults: A systematic review. Child and Adolescent Social Work Journal 29(3): 241-266.

5. Becker KD, Buckingham SL, Brandt NE (2015) Engaging youth and families in school mental health services. Child Adolesc Psychiatr Clin N Am 24(2): 385-398

6. Staudt M, Lodato G, Hickman CR (2012) Therapists talk about the engagement process. Community Ment Health J 48(2): 212-218.

7. Schwarzer R (2002) Health action process approach (HAPA) Gesundheitspsychologie von Abis Z. Hogrefe, Göttingen. 2002: 241-245.

8. Kraft P, Sutton SR, Reynolds HM (1999) The trans theoretical model of behaviour change: Are the stages qualitatively different? Psychology \& Health 14(3):433-450. 
9. Lequerica AH, Kortte K (2010) Therapeutic engagement: a proposed model of engagement in medical rehabilitation. Am J Phys Med Rehabil 89(5): 415-422.

10. Adika VO, Nzewi N, Apiyanteide FA (2011) Knowledge and opinion on stroke rehabilitation and outcome among stroke patients in Bayelsa State, Nigeria. International Journal of Tropical Medicine 6(4): 90-96.

11. Österberg SA, Baigi A, Bering C, Fridlund B (2010) Knowledge of heart disease risk in patients declining rehabilitation. Br J Nurs 19(5): 288293.

12. Blanchard C, Arthur HM, Gunn E (2015) Self-efficacy and outcome expectations in cardiac rehabilitation: Associations with women's physical activity. Rehabil Psychol 60(1): 59-66.

13. Hoffmann TC, Del Mar C (2015) Patients expectations of the benefits and harms of treatments, screening, and tests: systematic review. JAMA intern med 175(2): 274-286.

14. Schwarzer R (2014) Self-efficacy: Thought control of action. In: Schwarzer R (Ed.), Self-efficacy: Thought control of action. Taylor \& Francis, UK.

15. Dohnke B, Nowossadeck E, Müller-Fahrnow W (2010) Motivation and participation in a phase III cardiac rehabilitation programme: an application of the health action process approach. Res Sports Med 18(4): 219-235.

16. Saltapidas H, Ponsford J (2007) The influence of cultural background on motivation for and participation in rehabilitation and outcome following traumatic brain injury. J Head Trauma Rehabil 22(2): 132-139.

17. Kortte KB, Falk LD, Castillo RC, Johnson-Greene D, Wegener ST (2007) The Hopkins rehabilitation engagement rating scale: development and psychometric properties. Arch Phys Med Rehabil 88(7): 877-884.

18. Lequerica AH, Rapport LJ, Whitman RD, Scott R, Steven J, et al. (2006) Psychometric properties of the rehabilitation therapy engagement scale when used among individuals with acquired brain injury. Rehabilitation Psychology 51(4): 331-337.

19. Lenze EJ, Munin MC, Quear T, Dew MA, Rogers JC, et al. (2004) The Pittsburgh Rehabilitation Participation Scale: reliability and validity of a clinician-rated measure of participation in acute rehabilitation. Arch Phys Med Rehabil 85(3): 380-384.

20. Bright FA, Kayes NM, Worrall L, McPherson KM (2015) A conceptual review of engagement in healthcare and rehabilitation. Disabil Rehabil 37(8): 643-654

\section{Your subsequent submission with Crimson Publishers} will attain the below benefits

- High-level peer review and editorial services

- Freely accessible online immediately upon publication

- Authors retain the copyright to their work

- Licensing it under a Creative Commons license

- Visibility through different online platforms

- Global attainment for your research

- Article availability in different formats (Pdf, E-pub, Full Text)

- Endless customer service

- Reasonable Membership services

- Reprints availability upon request

- One step article tracking system 